\title{
The future history of the Solar System
}

\author{
The belief that the Sun will eventually mechanically engulf the orbit of the Earth may be misplaced, but even the \\ coming billion years could bring discomfort to the Earth's inhabitants.
}

WHEN will the Sun engulf the orbit of the Earth? That was one of a list of questions given, without further discussion, in this year's 125 th anniversary issue (Nature 372, $14 ; 1994)$ as illustrations of our continuing ignorance of the world we live in.

The origin of the question is familiar. The Sun is now a simple and stable astrophysical object, burning hydrogen as a thermonuclear fuel, but the time will come when the helium produced in that process will predominate over hydrogen in the core. Then energy production will be confined to a surrounding shell of hydrogen and the core will itself collapse. At that stage, the Sun will become a red giant star, its vastly extended envelope sustained by an increased flux of radiation, but the external temperature decreased so that it appears redder than now. Red giant stars comparable with the Sun in mass are known to have radii greater than the average radius of the Earth's orbit about the Sun. So is it not meaningful to ask when the Sun's radius will exceed that of the Earth's orbit?

A correspondent, Dr Dieter Hartmann from Clemson University in South Carolina, has now written modestly to say that, while the question is sensible, there is already a cogent answer in the literature. And that is that there is a good chance that the Sun will never engulf the Earth's orbit. The envelope will indeed be enlarged enormously, and the orbit of Mercury will almost certainly be engulfed. But long before that happens, the Sun may have lost up to a quarter of its mass and the Earth's orbit will have been enlarged as the Sun's gravitational attraction declines.

So everything will be all right with the Earth, then? The temptation to jump to that comfortable conclusion will be quickly exorcised by a glance at the article to which Hartmann refers, which is an attempt to chart in detail the behaviour of the Sun over the 10 billion years or more ahead.

The authors are members of a group linked with W. A. Fowler at the California Institute of Technology and comprise I. Juliana Sackmann and Kathleen E. Kraemer from that institution (but the latter has moved to Boston University) and Arnold I. Boothroyd from the University of Toronto (Astrophys. J. 418. 457-468; 1993). Their future history of the Sun is the product of a stellar model incorporating the known idiosyncrasies of the Sun, its chemical composition for example.

Predicting the future convincingly requires a demonstration that past history can

NATURE · VOL 372 - 15 DECEMBER 1994 be accurately reconstructed. Sackmann and her colleagues are explicit about their assumptions. The age of the meteorites of 4.55 billion years estimated by $\mathrm{G}$. Wasserburg and his colleagues (also at Caltech) presumably marks a point when the early Sun still had a primitive accretion disk, and consisted of a glowing mass of gas and dust whose energy was derived exclusively from gravitational collapse.

That phase, for the Sun, would have occupied no more than 50 million years. For the first 10 million years, as the nebula shrank, the external temperature would have been roughly constant (and roughly $1300 \mathrm{~K}$ less than at present) and the luminosity would have declined with the surface area, perhaps by a factor of 30. A further 40 million years would have been occupied in further (and slower) gravitational collapse before hydrogen burning in the core began.

Critical to this model of the Sun are the assumptions made about the original chemical composition of the solar nebula. The raw material of the Sun, consisting as it does of material recycled through earlier generations of stars, contains elements heavier than oxygen produced in supernova explosions, as well as a greater abundance of ${ }^{4} \mathrm{He}$ than there would have been in the primordial material. Sackmann and her colleagues choose a helium abundance $(0.274)$ that fits with the present luminosity and radius of the Sun. They say that their present use of their solar model is likely to be an improvement on earlier uses of it because they have had the benefit of improved (and higher) estimates of the opacity of the molecular constituents of the outer envelope of the Sun, materials such as CO. The opacity of the stellar material is crucial to determining the extent to which outward radiation pressure sustains the outer regions of the Sun.

What, on this view, is the Sun like at present? The central temperature is $\mathbf{1 5 . 4 3}$ million degrees and the central density 145.7 $\mathrm{g} \mathrm{cm}^{-3}$. Interestingly, after just over 4.5 billion years of hydrogen-burning, the mass fraction of hydrogen at the very centre of the Sun has already fallen by almost a half, from 0.7064 to 0.3632 . The radius at which convection takes over from radiative transfer in the outward flux of energy in the Sun is reckoned, from the model, to be 0.741 of the solar radius, at which level the temperature is still nearly 2 million degrees $\mathrm{K}$. Amazingly as always, the fraction of the Sun's mass in this outer convective layer is a mere 1.68 per cent.

And what of the future, as the mass- fraction of hydrogen at the centre continues to decline? Hydrogen will remain the thermonuclear fuel for the Sun for a long time to come, perhaps for a further 6.4 billion years. But this quiescent phase will not be free from discomfort for those living on the Earth. Over the coming 3 billion years, the luminosity of the Sun will gradually increase by 33 per cent, the external temperature will marginally increase and the radius of the Sun will grow by 13 per cent.

Long before that condition is reached, the Sun's luminosity will have become intolerable. Indeed, there are some who argue that a mere 10 per cent increase of the Sun's luminosity, likely in the next 600 million years, would evaporate surface water. The oceans would disappear when the Sun's luminosity had increased by 40 per cent, perhaps 3.5 billion years from now. Relatively, solar catastrophe is not far away.

Solar cataclysm is not expected until much later. Some 4.8 billion years from now, hydrogen-burning in the core will be halted, and a shell of burning hydrogen enclosing a quiescent core of helium and heavier elements will become thicker over the succeeding 1.6 billion years (taking the luminosity to 2.2 times its present value). That is when the core begins to collapse and when, 6.5 billion years ahead, the Sun's envelope expands rapidly, over 700 million years, to a radius ten times the present.

That is when the fun and games will begin. Now a true red giant, the Sun's radius and luminosity will both increase, the temperature at the centre will have reached 100 million degrees, and helium-burning will eventually begin in a shell surrounding the core. The result of that will be to carry the envelope of the Sun out to the present orbit of Venus, but because the Sun is reckoned to lose 28 per cent of its mass during its red giant phase, even Venus will not be directly engulfed, for the radius of its orbit (inversely proportional to the mass of the Sun) will have enlarged to keep the planet out of range.

The Earth, 38 per cent farther away than at present from the centre of the Sun, will be mechanically safe, but very little imagination is required to guess the effect on its surface of a Sun whose peak luminosity is 2,349 times what it is at present and which subtends an angle of 69 degrees at the Earth's surface. There is worse to follow. In a mere 160 million years, there are five further helium flashes, whereupon the Sun settles down to be a white dwarf and Sackmann and her colleagues "terminate" their calculations. As well they might. John Maddox 